\title{
False vacuum decay in de Sitter space-time
}

\author{
V. A. Rubakov, S. M. Sibiryakov \\ Institute for Nuclear Research of the Russian Aademy of Sciences, \\ 117312, Moscow, 60th October Anniversary Prospect, 7a
}

\begin{abstract}
We suggest a technique that explicitly accounts for the structure of an initial state of quantum field in the semiclassical calculations of path integral in curved space-time, and consider decay of metastable state (conformal vacuum of scalar particles above false classical vacuum) in background de Sitter spacetime as an example. Making use of this technique, we justify the Coleman-De Luccia approach to the calculation of the decay probability. We propose an interpretation of the Hawking-Moss instanton as a limiting case of constrained instantons. We find that an inverse process of the transition from true vacuum to false one is allowed in de Sitter space-time, and calculate the corresponding probability.
\end{abstract}

\section{Introduction}

The description of false vacuum decay in quantum field theory is of considerable interest, especially in view of the developments of inflationary theories and models that explain the baryon asymmetry of the Universe by invoking first order phase transitions. It was established some time ago that the process of tunneling from the false vacuum to the true one is semiclassical in weakly coupled theories, and the adequate technique was developed for the calculation of the decay probability in scalar field theories in Minkowski space-time [1, 22, 3]. It was shown that the decay proceeds through the materialization of bubbles of the new phase (true vacuum) in the metastable one (false vacuum), and that the exponent in the semiclassical expression 
for the probability of the formation of a bubble per unit time per unit volume,

$$
\Gamma=A \mathrm{e}^{-B}
$$

is determined by a solution to the classical field equations in Euclidean space-time with the boundary conditions that require that the field tends to the false vacuum as the Eucliden time $\tau$ approaches $\pm \infty$. The exponent $B$ is equal to the Euclidean action calculated on the non-trivial (not identically equal to the false vacuum) solution of this boundary problem, minus the action on the trivial solution. If the boundary problem has several non-trivial solutions, then the value of $B$ is determined by the least action one. It was shown that for any scalar potential there exists an $O(4)$ symmetric solution (bounce) [3], and that it has the smallest action among non-trivial solutions [4]. The analytical continuation of this solution to pure imaginary $\tau, \tau=i t$, gives the configuration of the bubble after its materialization as the function of the physical time $t$. These results were obtained by making use of the path integral technique. It is worth noting (although in this paper we will consider leading semiclassical exponents only) that this technique allows one to calculate pre-exponential factor $A$ as well [3].

The problem of accounting for gravitational effects in the formation and subsequent evolution of bubbles was studied by Coleman and De Luccia [5]. They proposed, by analogy to the case of flat space-time, that the probability of the formation of a bubble is determined by an $O(4)$-symmetric solution to the system of equations consisting of the Klein-Gordon-Fock equation and Einsten equations for the scalar field and Riemannian metrics with Euclidean signature. The exponent $B$ is again equal to the difference between the actions of non-trivial and trivial solutions of this system (the trivial solution is such that the scalar field is identically equal to its false vacuum value), where the action contains now two terms: the action of the scalar field in curved space-time and the action of the gravitational field. It has been shown [6], that if the scalar potential is non-negative (this case is most interesting for inflationary theories), then the metrics of this solution describes the space with the topology of a four-sphere $S^{4}$. The analytical continuation of this solution to the space-time with Minkowski signature gives the evolution of the bubble in real space-time; the space-time in distant past is described by the de Sitter metrics.

We think that this appealing and simple approach needs further justification. In the first place, it is based solely on the analogy to field theory in flat space-time. This analogy may in principle not work for space-times of large curvature. In particular, 
for large class of scalar potentials, the only $O(4)$-symmetric Euclidean solution is the Hawking-Moss instanton [7], which is a four-sphere with constant scalar field. This instanton differs considerably from the bounce of Minkowskian theory, and its naive analytical continuation to space-time with Minkowskian signature would correspond to homogeneous scalar field rather than to the bubble of the true vacuum in the false one.

Moreover, the very notion of the false vacuum in curved space-time needs qualification. Besides specifying the classical vacuum - the average value of the scalar field - one has also to specify the state of quantum fluctuations of the field. Indeed, the vacuum state is not uniquely defined in curved space-time even in the theory of free quantum fields, as it depends on the choice of modes in which the field operators are decomposed (see, e.g., refs. [8, 9]). In this regard, it is of interest to establish what choice of quantum vacuum leads to Coleman-De Luccia and Hawking-Moss prescriptions. 円

In a certain range of the parameters of the theory, part of these problems may be analyzed by making use of the stochastic approach [11]. Within this approach, it has been demonstrated [11, 12] that the Hawking-Moss instanton action indeed coincides with the exponent for the probability of the decay of the classical false vacuum, if the state of quantum fluctuations above this vacuum is the conformal vacuum of the scalar field. However, the role of the Hawking-Moss solution itself remains unclear within the stochastic approach.

A natural step in clarifying the above points is to study the false vacuum decay in the limiting case when the characteristic scale of variation of the scalar potential during the tunneling process is small compared to the potential itself. In this case one can neglect the changes of metrics in the process of the bubble formation, so one arrives at field theory in curved background space-time, namely, in the de Sitter background. An obvious advantage of this approximation is that one does not have to deal with quantum gravity effects, i.e., the problem may be analysed in terms of ordinary field theory, albeit in curved space-time. We will restrict our analysis to the choice of the quantum state above the classical false vacuum as the conformal vacuum of scalar particles; this state appears naturally in inflationary theories. Under these

\footnotetext{
${ }^{1}$ It is likely that different choices of quantum states above a given classical false vacuum will give rise to considerably different decay probabilities. Indeed, there often emerges the notion of the effective temperature that characterizes one or another vacuum already in the theory of free fields (see, e.g., ref. [9]). On the other hand, the exponent for the decay of the metastable state strongly depends on temperature in Minkowski space-time [10].
} 
assumptions we will show that the decay of the metastable state is indeed described by the Coleman-De Luccia instanton (in models where it exists), give the interpretation of the Hawking-Moss instanton as the limiting case of constrained instantons, and discuss solutions that do not have analogs in flat space-time and describe tunneling from the true vacuum to the false one.

\section{Preliminaries}

We consider the theory of the scalar field $\phi$ with the potential shown in fig. 1. The

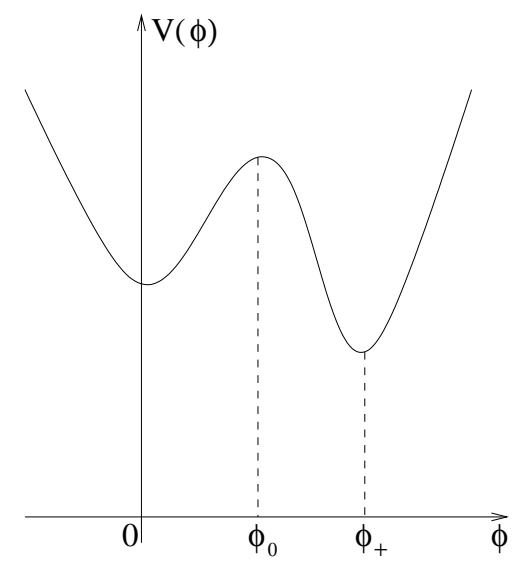

Figure 1:

states $\phi=0$ and $\phi=\phi_{+}$correspond to the false and true vacua, respectively; they are separated by the local maximum of the scalar potential, $V\left(\phi_{0}\right)$. Let us first discuss the following question: does it make sense at all to consider gravitational effects on tunneling in the background metrics approximation? Let us introduce the mass scale $M$ that measures the variation of the potential in the interval between the two vacua. The gravitational field is characterized by the Hubble parameter $H$. The gravitational effects are important for the formation of the bubble when the ratio $\frac{H}{M}$ is of order or larger than 1, while the approximation of background de Sitter metrics is valid at

$$
\frac{M^{4}}{V\left(\phi_{+}\right)} \ll 1
$$

Making use of the Einstein equation,

$$
H^{2}=\frac{8 \pi}{3 M_{P l}^{2}} V\left(\phi_{+}\right)
$$


one finds

$$
\frac{H}{M}=\frac{1}{M M_{P l}} \sqrt{\frac{8 \pi V\left(\phi_{+}\right)}{3}}
$$

This ratio can easily be of order one, if $\frac{V\left(\phi_{+}\right)^{\frac{1}{4}}}{M_{P l}} \gtrsim \frac{M}{V\left(\phi_{+}\right)^{\frac{1}{4}}}$. At the same time, the inequality (1) may well be satisfied, and the energy density $V\left(\phi_{+}\right)$may be small compared to the Planck value. Hence, the approximation of background metrics is legitimate in some region of parameters, and in the same region the gravity effects are not negligible.

In what follows we will consider the scalar field in the background metrics, so we may freely change the origin of its energy. It is convenient to set $V(0)=0$, as shown in fig. 2.

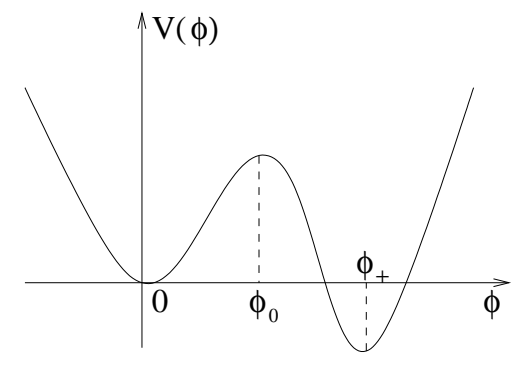

Figure 2:

The choice of the state of quantum fluctuations above the classical false vacuum is related to the choice of reference frame. We will use the frame with flat surfaces of constant time, in which the de Sitter metrics takes the following form,

$$
d l^{2}=\frac{1}{H^{2} \eta^{2}}\left(d \eta^{2}-d x_{i} d x_{i}\right)
$$

The conformal time parameter $\eta$ changes from $-\infty$ to 0 ; it is related to the sinchronous time $t$ as $\eta=-\frac{1}{H} e^{-H t}$. This coordinate system covers only one half of the full de Sitter space (see, e.g., ref. [13]), but it is this part that is of interest to inflationary models. The action of the model in these coordinates is

$$
S=\int \frac{d^{3} x d \eta}{H^{4} \eta^{4}}\left[\frac{H^{2} \eta^{2}}{2}\left(\left(\frac{\partial \phi}{\partial \eta}\right)^{2}-\left(\frac{\partial \phi}{\partial x^{i}}\right)^{2}\right)-V(\phi)\right]
$$

Let us decompose the quantum perturbations about the false vacuum in scalar modes which are natural for this frame [9],

$$
\hat{\phi}(\mathbf{x}, \eta)=\int \frac{d^{3} k}{(2 \pi)^{\frac{3}{2}}} H \eta\left(a_{k} \chi_{k}(\eta)+a_{-k}^{+} \chi_{k}^{*}(\eta)\right) e^{i \mathbf{k x}}
$$




$$
\chi_{k}(\eta)=\frac{(\pi \eta)^{\frac{1}{2}}}{2} H_{\nu}^{(2)}(k \eta), \quad \nu^{2}=\frac{9}{4}-\frac{m^{2}}{H^{2}}, \quad m^{2}=V^{\prime \prime}(0), \quad k=|\mathbf{k}|
$$

Here $H_{\nu}$ are the Hankel functions. The conformal vacuum above the classical false one is a state that is annihilated by all operators $a_{k}: a_{k}|0\rangle=0$. Though this definition makes sense in perturbation theory only, it will be sufficient for our purposes. Our choice of frame, and related choice of quantum vacuum is due to several reasons:

1. This frame and this definition of vacuum appear naturally in inflationary theories (see., e.g., ref. 114).

2. This vacuum is invariant under spatial translations, $x \rightarrow x+a$, and scale transformations, $\eta \rightarrow \alpha \eta, x \rightarrow \alpha x$, which are the global isometries of the half of the de Sitter space.

3. The modes $\chi_{k}$ reduce to negative frequency plane waves at large negative $\eta$,

$$
\chi_{k} \rightarrow \frac{1}{(2 k)^{\frac{1}{2}}} e^{-i k \eta}, \quad \eta \rightarrow \infty
$$

So, one expects that at large negative times the theory in coordinates $(\eta, \mathbf{x})$ is similar to the theory in flat space-time, and this is indeed the case (see below).

The false vacuum state, as defined above, is metastable. The problem is to find the leading semiclassical exponent for the probability of its decay.

\section{Classical problem and boundary conditions}

In this Section we show that in the semiclassical regime, the exponent for the probability of the false vacuum decay is indeed determined by a certain classical solution to the scalar field equation, and obtain the boundary conditions that are to be imposed on the solution.

Let us introduce the following notations: $|n, \eta\rangle$ is a state $n$ at time $\eta$ (we work in the Heisenberg representation); in particular, $|0, \eta\rangle$ is the state of the false vacuum at time $\eta,|\phi(\mathbf{x}), \eta\rangle$ is the common eigenstate of the field operators taken at time

$\eta$. To calculate the probability of the false vacuum decay, we will need the matrix element $\left\langle\phi_{f}\left(\mathbf{x}, \eta_{f}\right) \mid 0, \eta_{i}\right\rangle$, where $\eta_{f}$ and $\eta_{i}$ are the final and initial moments of time, respectively. This matrix element can be written in terms of path integral,

$$
\left\langle\phi_{f}\left(\mathbf{x}, \eta_{f}\right) \mid 0, \eta_{i}\right\rangle=\int D\left[\phi_{i}(\mathbf{x})\right]\left\langle\phi_{i}(\mathbf{x}), \eta_{i} \mid 0, \eta_{i}\right\rangle \int D[\phi(\mathbf{x}, \eta)] e^{i S[\phi]}
$$


Here $S[\phi]$ is the action in the interval $\left(\eta_{i}, \eta_{f}\right)$ evaluated on the field configuration $\phi(\mathbf{x}, \eta)$, obeying the boundary conditions

$$
\phi\left(\mathbf{x}, \eta_{i}\right)=\phi_{i}(\mathbf{x}), \quad \phi\left(\mathbf{x}, \eta_{f}\right)=\phi_{f}(\mathbf{x})
$$

The outer integral is over the initial configurations of the field. Two comments are in order:

1. Time interval $\left(\eta_{i}, \eta_{f}\right)$ is taken to be much larger than any time scale of the theory. We are ultimately interested in the limit $\eta_{i} \rightarrow-\infty$.

2. Until now the variables $\mathbf{x}$ and $\eta$ were real coordinates in the de Sitter spacetime. However, one can consider the space of complex $(\mathbf{x}, \eta)$ and analytical continuations of the metrics and field into this space. The four-dimensional hypersurface over which the action in eq. (5) is integrated, may be deformed arbitrarily in this space, provided that its boundaries, the three-dimensional initial and final surfaces, remain intact.

The decay width of the false vacuum takes the following form,

$$
\Gamma=\int D\left[\phi_{f}(\mathbf{x})\right]\left|\left\langle\phi_{f}, \eta_{f} \mid 0, \eta_{i}\right\rangle\right|^{2}
$$

where the integration runs over the configurations $\phi_{f}(\mathbf{x})$ that are close to the true vacuum (the meaning of the latter requirement will become clear later on). Upon substituting eq. (5) into eq. (6) one gets

$$
\begin{aligned}
\Gamma & =\int D\left[\phi_{f}(\mathbf{x})\right] \int D\left[\phi_{1 i}(\mathbf{x})\right] D\left[\phi_{2 i}^{*}(\mathbf{x})\right]\left\langle\phi_{1 i}, \eta_{i} \mid 0, \eta_{i}\right\rangle\left\langle\phi_{2 i}, \eta_{i} \mid 0, \eta_{i}\right\rangle^{*} \\
& \times \int D\left[\phi_{1}(\mathbf{x}, \eta)\right] D\left[\phi_{2}(\mathbf{x}, \eta)\right] e^{i\left(S\left[\phi_{1}\right]-S^{*}\left[\phi_{2}\right]\right)}
\end{aligned}
$$

Our immediate purpose is to evaluate the path integrals in eq. (元) by the saddle point technique (which of course assumes that the problem is semiclassical). Let us first integrate over $D\left[\phi_{1}\right] D\left[\phi_{2}\right]$. The saddle point of the integrand is a solution of the classical field equations. The corresponding classical solutions $\phi_{1}(\mathbf{x}, \eta)$ and $\phi_{2}(\mathbf{x}, \eta)$ obey the following conditions,

$$
\phi_{1}\left(\mathbf{x}, \eta_{i}\right)=\phi_{1 i}(\mathbf{x}), \quad \phi_{2}\left(\mathbf{x}, \eta_{i}\right)=\phi_{2 i}(\mathbf{x}), \quad \phi_{1}\left(\mathbf{x}, \eta_{f}\right)=\phi_{2}\left(\mathbf{x}, \eta_{f}\right)=\phi_{f}(\mathbf{x})
$$

To integrate over $D\left[\phi_{1 i}\right] D\left[\phi_{2 i}\right]$, one needs explicit expressions for the matrix elements of the type $\left\langle\phi_{i}(\mathbf{x}), \eta_{i} \mid 0, \eta_{i}\right\rangle$. Their calculation parallels the case of flat space-time (this 
is due to the fact that the modes $\chi_{k}$ tend to plane waves as $\left.\eta \rightarrow-\infty\right)$. Since the relevant matrix elements are large only for field configurations close to the false vacuum, one makes use of perturbation theory. Let us introduce the momenta conjugate to the field operators,

$$
\begin{aligned}
\hat{\pi}(\mathbf{x}, \eta) & =\frac{1}{H^{2} \eta^{2}} \frac{\partial \hat{\phi}}{\partial \eta} \\
& =\int \frac{d^{3} k}{(2 \pi)^{\frac{3}{2}}} \frac{1}{H^{2} \eta^{2}}\left(a_{k} \chi_{k}(\eta)+a_{-k}^{+} \chi_{k}^{*}(\eta)\right) e^{i \mathbf{k x}} \\
& +\int \frac{d^{3} k}{(2 \pi)^{\frac{3}{2}}} \frac{1}{H \eta}\left(a_{k} \dot{\chi}_{k}(\eta)+a_{-k}^{+} \dot{\chi}_{k}^{*}(\eta)\right) e^{i \mathbf{k x}}
\end{aligned}
$$

Note that the first term in eq. (9) is negligible at large negative $\eta$. By introducing the spatial Fourier components,

$$
\hat{\phi}\left(\mathbf{k}, \eta_{i}\right)=H \eta_{i}\left(a_{k} \chi_{k}\left(\eta_{i}\right)+a_{-k}^{+} \chi_{k}^{*}\left(\eta_{i}\right)\right), \quad \hat{\pi}\left(\mathbf{k}, \eta_{i}\right)=\frac{1}{H \eta}\left(a_{k} \dot{\chi}_{k}\left(\eta_{i}\right)+a_{-k}^{+} \dot{\chi}_{k}^{*}\left(\eta_{i}\right)\right)
$$

one obtains the standard commutational relations

$$
\left[\hat{\pi}\left(-\mathbf{k}, \eta_{i}\right), \hat{\phi}\left(\mathbf{q}, \eta_{i}\right)\right]=-i \delta(\mathbf{k}-\mathbf{q})
$$

Hence, the operators $\hat{\phi}\left(\mathbf{k}, \eta_{i}\right)$ and $\hat{\pi}\left(\mathbf{k}, \eta_{i}\right)$ may be realized as multiplication by $\phi(\mathbf{k})$ and variational derivation, $\frac{-i \delta}{\delta \phi(-\mathbf{k})}$, respectively. In this way one arrives at the coordinate representation. The state $\left|0, \eta_{i}\right\rangle$ in this representation is the functional $F[\phi(\mathbf{k})]=\left\langle\phi(\mathbf{k}), \eta_{i} \mid 0, \eta_{i}\right\rangle$. One obtains the equation for this functional by exressing the operators $a_{k}$ through $\hat{\phi}\left(\mathbf{k}, \eta_{i}\right)$ and $\hat{\pi}\left(\mathbf{k}, \eta_{i}\right)$ and recalling that $a_{k}$ annihilate the vacuum,

$$
\left(\frac{\delta}{\delta \phi(-\mathbf{k})}+\frac{k}{\left(H \eta_{i}\right)^{2}} \phi(\mathbf{k})\right) F=0
$$

(to obtain eq. (10) one makes use of the asymptotics (4)). Hence,

$$
F=F_{0} \exp \left[-\int \frac{k \phi(\mathbf{k}) \phi(-\mathbf{k})}{2\left(H \eta_{i}\right)^{2}} d^{3} k\right]
$$

The normalization constant $F_{0}$ will be inessential for our purposes.

Let us substitute eq. (11) into eq. (7) and integrate over $D\left[\phi_{1 i}\right]$. The saddle point of the integrand is determined by the condition

$$
\frac{\delta}{\delta \phi_{1 i}(-\mathbf{k})}\left(-\int \frac{k \phi_{1 i}(\mathbf{k}) \phi_{1 i}(-\mathbf{k})}{2\left(H \eta_{i}\right)^{2}} d^{3} k+i S\left[\phi_{1}\right]\right)=0
$$


which implies that

$$
\frac{k}{\left(H \eta_{i}\right)^{2}} \phi_{1 i}(\mathbf{k})+i \frac{1}{\left(H \eta_{i}\right)^{2}} \dot{\phi}_{1}\left(\mathbf{k}, \eta_{i}\right)=0
$$

In this way we obtain the positive frequency condition at $\eta \rightarrow-\infty$ that has to be imposed on the classical solution $\phi_{1}$. The same argument leads to the positive frequency condition on $\phi_{2}$ at large negative $\eta$. The boundary conditions for $\phi_{1}$ and $\phi_{2}$ at $\eta_{f}$ are also the same (see eq. (8)); furthermore, $\phi_{1}$ and $\phi_{2}$ are solutions to the same classical field equation. So, $\phi_{1}$ and $\phi_{2}$ are actually one and the same function f which we denote merely by $\phi$. Thus, the saddle point integration over the intermediate and initial values of the field gives

$$
\Gamma \propto \int D\left[\phi_{f}\right] e^{i\left(S[\phi]-S^{*}[\phi]\right)}
$$

where $\phi(\mathbf{x}, \eta)$ is (in general, complex) solution of the classical scalar field equation in the de Sitter background metrics, obeying the boundary conditions

$$
\phi\left(\mathbf{x}, \eta_{f}\right)=\phi_{f}(\mathbf{x})
$$

$\phi \quad$ contains only positive frequency waves at $\eta \rightarrow-\infty$

As we are going to discuss the leading semiclassical exponent only, the pre-exponential factor is not shown in eq. (12). Note that the boundary condition (13), in similarity to Minkowski background, allows one not only to deform the integration contour but also to shift the boundary "point" $\eta_{i}=-\infty$ in upper complex half-plane of the variable $\eta$. Hereafter the integration contour (or simply contour) means the hypersurface used in the calculation of the action in eq. (12).

There still remains one integration in eq. (12). We find the corresponding saddle point equation:

$$
\dot{\phi}\left(\mathbf{x}, \eta_{f}\right)-\dot{\phi}^{*}\left(\mathbf{x}, \eta_{f}\right)=0
$$

which, together with reality of $\phi_{f}(\mathbf{x})$ gives the following condition,

$$
\phi(\mathbf{x}, \eta) \text { is real at } \eta>\eta_{f}
$$

When the solution $\phi(\eta)$ (we temporarily ignore the dependence of $\phi$ on spatial coordinates) is continued along the real axis from the domain $\eta>\eta_{f}$ to the region $\eta<\eta_{f}$, it

\footnotetext{
${ }^{2}$ Indeed, a general solution to the second order equation contains two arbitrary complex functions of three variables. The positive frequency condition kills one of these degrees of freedom. The second function is determined by the boundary condition imposed at the final time.
} 
will remain real. This property may seem to contradict the condition (13). However, this observaion only implies that a solution with required properties does not exist on the real axis, so one has to search for such a solution on the deformed contour that avoids singular points of the function $\phi$ (cf. ref. [15]).

The values of $\phi$ in upper the and lower half-planes are related by $\phi\left(\eta^{*}\right)=\phi^{*}(\eta)$. Taking this property into account, we rewrite the exponent in eq. (12) as follows,

$$
i\left(S[\phi]-S^{*}[\phi]\right)=i\left(S_{C}[\phi]-S_{C^{*}}[\phi]\right)=i S_{C+C^{*}}[\phi]
$$

Here $S_{C}, S_{C^{*}}$ and $S_{C+C^{*}}$ stand for the values of the action at the contours $C$, its conjugate contour $C^{*}$ and their sum (see fig. 3). Now we can relax the condition that the contours $C$ and $C^{*}$ meet at the point $\eta_{f}$ and deform the contour $C+C^{*}$ into a contour $C_{0}$ that consists of the imaginary axis and infinitesimal semi-circle around the singular point of the metrics, $\eta=0$.

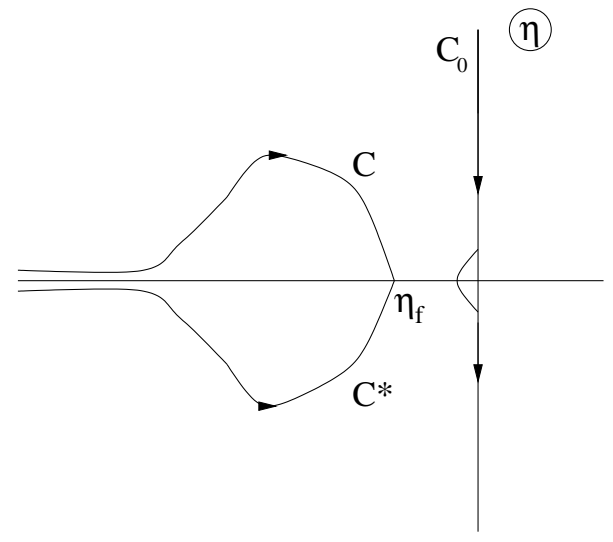

Figure 3:

It is convenient to introduce a new coordinate $\zeta$ such that $\eta=i \zeta$, and rewrite the action in the follwing form,

$$
i S_{C_{0}}[\phi]=\int d^{3} x \int_{-\infty}^{\infty} d \zeta \frac{1}{H^{4} \zeta^{4}}\left[\frac{H^{2} \zeta^{2}}{2}\left(\left(\frac{\partial \phi}{\partial \zeta}\right)^{2}+\left(\frac{\partial \phi}{\partial x^{i}}\right)^{2}\right)-V(\phi)\right] \equiv-S_{\zeta}[\phi]
$$

(Primed integral denotes the integration near $\zeta=0$ along the contour $C_{0}$.)

Let us summarize the outcome of the analysis made in this Section.

1. The leading semiclassical exponent of the false vacuum decay may be written as follows,

$$
\Gamma \propto e^{-S_{\zeta}[\phi]}
$$


2. The function $\phi$ in eq. (16) is a classical solution to the field equation at the contour $C_{0}$.

3. The analytical continuation of $\phi$ to the real values of $\eta$ should be real.

4. $\phi(\zeta)$ tends to zero as $\zeta \rightarrow \infty$ (the latter condition is a consequence of eq. (13)).

To deform the integration contour (four-dimensional hypersurface in the space of complex $\mathbf{x}$ and $\eta$ ) further, it is useful to discuss the analytical continuations of the de Sitter metrics.

\section{Analytical continuation of metrics}

In the previous Section we introduced the coordinate $\zeta$ related to $\eta$ by $\eta=i \zeta$. Let us first analyze the metrics of space with coordinates $(\mathbf{x}, \zeta)$ at $0<\zeta<\infty$. It is clear from eq.(2) that the line element in this space is

$$
d l^{2}=\frac{d \zeta^{2}+d x_{i}^{2}}{H^{2} \zeta^{2}}
$$

that is, the metrics has Euclidean signature. This is the space of constant negative curvature, as is clear directly from the corresponding property of the de Sitter spacetime [9]. However, it can be shown that this space is geodesically complete, unlike the half of the de Sitter space that has been considered above.

Let us introduce spherical coordinates in three-dimensional space with coordinates $x_{i}$; then eq. (17) is written as follows,

$$
d l^{2}=\frac{d \zeta^{2}+d \rho^{2}+\left(d \Omega_{2}\right)^{2}}{H^{2} \zeta^{2}}
$$

where $\rho=\sqrt{x_{1}^{2}+x_{2}^{2}+x_{3}^{2}}$, and $\left(d \Omega_{2}\right)^{2}=d \theta^{2}+\sin \theta^{2} d \varphi^{2}, \quad 0 \leq \theta \leq \pi, \quad 0 \leq \varphi<2 \pi$ is the line element on unit two-dimensional sphere. Until now, the coordinates $x, \eta, \zeta, \rho$ had the dimension of length. However, it is convenient to make these coordinates dimensionless, i.e., measure them in units of the inverse Hubble parameter. The line element is not changed after this redefinition, while the expression for the action changes only slightly,

$$
S_{\zeta}=-\int d^{3} x \int_{-\infty}^{\infty} d \zeta \frac{1}{\zeta^{4}}\left[\frac{\zeta^{2}}{2 H^{2}}\left(\left(\frac{\partial \phi}{\partial \zeta}\right)^{2}+\left(\frac{\partial \phi}{\partial x^{i}}\right)^{2}\right)-\frac{V(\phi)}{H^{4}}\right]
$$


Let us introduce, in the space under discussion, the coordinates that are reminiscent of the usual spherical coordinates. Namely, let us choose a point (call it the origin) with coordinates $\rho=0, \zeta=\zeta_{0}$, and consider all possible geodesics that start from this point. The distance along these geodesics will be our radial coordinate $s$. Explicitly,

$$
s=\int_{\left(0, \zeta_{0}\right)}^{(\rho, \zeta)} d l=\int_{\left(0, \zeta_{0}\right)}^{(\rho, \zeta)} \frac{d \zeta^{\prime}}{\zeta^{\prime}} \sqrt{1+\left(\frac{d \rho}{d \zeta^{\prime}}\right)^{2}}
$$

The integration here is performed along the geodesics that connects the points $(\rho, \zeta)$ and $(0, \zeta)$, the derivative $\frac{d \rho}{d \zeta^{\prime}}$ is understood correspondingly. Note that the value of $\zeta_{0}$ is actually unimportant, as its change is equivalent to the change of scale. In what follows we set it equal to 1 for definiteness. Straigtforward but tedious calculations lead to the following formulae that express the coordinates $\rho$ and $\zeta$ through $s$ and an angular variable $\psi$ characterizing the direction of the relevant geodesics,

$$
\zeta=\frac{1}{\operatorname{ch} s-\cos \psi \operatorname{sh} s}, \quad \rho=\frac{\sin \psi \operatorname{sh} s}{\operatorname{ch} s-\cos \psi \operatorname{sh} s}, \quad 0 \leq s<\infty, \quad 0 \leq \psi \leq \pi
$$

Lines of constant $s$ and $\psi$ are shown in fig. 因. The line element in the new coordinates is

$$
d l^{2}=\frac{1}{H^{2}}\left(d s^{2}+\operatorname{sh}^{2} s\left(d \psi^{2}+\sin \psi^{2}\left(d \Omega_{2}\right)^{2}\right)\right)
$$

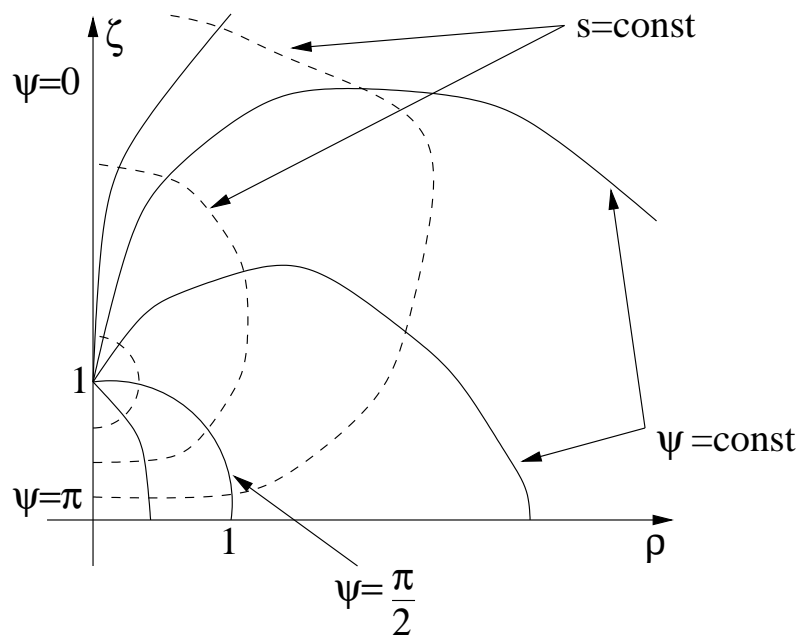

Figure 4:

Let us discuss the change of coordinates (20) in more detail. Equation $\psi=0$ describes a ray $\rho=0,1 \leq \zeta<\infty$ on the plane $(\rho, \zeta)$ while equation $\psi=\pi$ corresponds 
to the interval $\rho=0,0<\zeta \leq 1$. The line $\psi=\frac{\pi}{2}$ is a part of a circle $\rho^{2}+\zeta^{2}=1$. If one sends $s$ to infinity keeping $\psi \neq 0$ fixed, then

$$
\zeta \sim \frac{e^{-s}}{1-\cos \psi}, \quad \rho \rightarrow \frac{\sin \psi}{1-\cos \psi}, \quad s \rightarrow \infty
$$

Finally, the asymptotics $s \rightarrow \infty, \psi \sim e^{-2 s}$ corresponds to $\rho \rightarrow$ const, $\zeta \rightarrow \infty$.

Let us now consider $s$ as a complex variable (while keeping $\psi$ real) and analyse the corresponding analytical continuations of the metrics. The following cases will be of interest for our purposes:

1. $s=-i \pi+\hat{s}$, where $\hat{s}$ is real. Upon subsituting this relation into eq. (20) we find that $\rho$ is expressed through $\hat{s}$ in the same way as it has been expressed throgh $s$, and the formula for $\zeta$ changes by the sign only. Hence, these values of $s$ describe the space with negative $\zeta$; the structure of this space is completely analogous to the case of positive $\zeta$.

2. $s=-i \frac{\pi}{2}+s^{\prime}$, where $s^{\prime}$ is real. In this case one has

$$
\rho=\frac{\sin \psi \operatorname{ch} s^{\prime}}{\operatorname{sh} s^{\prime}-\cos \psi \operatorname{ch} s^{\prime}}, \quad \zeta=\frac{i}{\operatorname{sh} s^{\prime}-\cos \psi \operatorname{ch} s^{\prime}}
$$

Under the restriction $\cos \psi<$ th $s^{\prime}$ these formulae describe space with $\rho>0$ and pure imaginary $\zeta$ that changes from 0 to $+i \infty$. This space is nothing but half of the de Sitter space-time discussed in Sections 2 and 3 (the restriction imposed on $s^{\prime}$ at given $\psi$ is of course related to the geodesic incompleteness of this part of the de Sitter space). Lines of constant $s^{\prime}, \psi$ on the plane $\eta=i \zeta, \rho$ have the form shown in fig. 5. This space is characterized by the metrics

$$
d l^{2}=\frac{1}{H^{2}}\left(d s^{\prime 2}-\operatorname{ch}^{2} s^{\prime}\left(d \psi^{2}+\sin ^{2} \psi\left(d \Omega_{2}\right)^{2}\right)\right)
$$

3. $s=-i \sigma, \sigma$ is real and belongs to $[0, \pi]$. In terms of $\sigma$ the metrics (21) is

$$
d l^{2}=\frac{1}{H^{2}}\left(-d \sigma^{2}-\sin ^{2} \sigma\left(d \psi^{2}+\sin ^{2} \psi\left(d \Omega_{2}\right)^{2}\right)\right)
$$

which is the metrics of four-sphere (up to sign).

\section{Coleman-De Luccia solution}

In the approximation of background (non-dynamical) metrics, the Coleman-De Luccia configuration is the solution to the scalar field equation on four-sphere. In this 


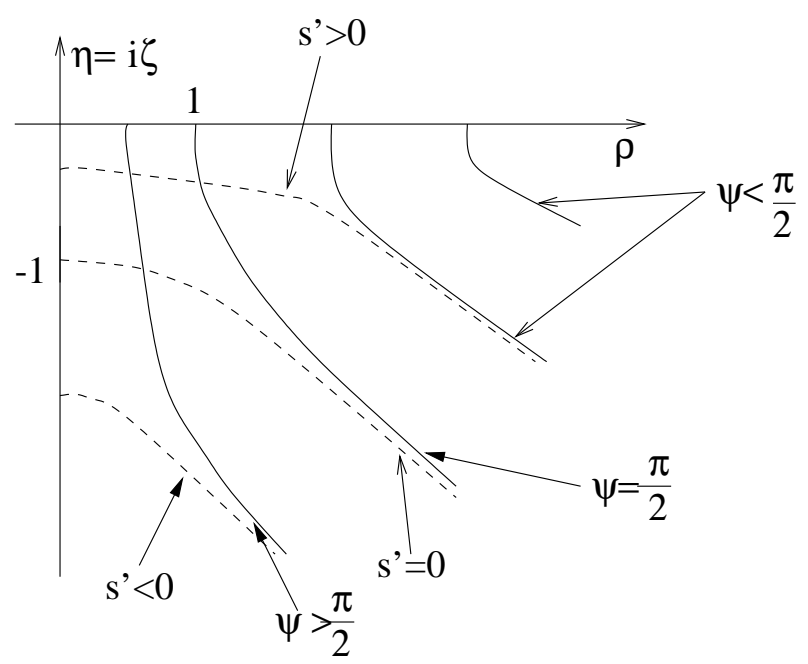

Figure 5:

Section we show that this configuration is indeed a solution to the boundary problem formulated in Section 3.

Let us rewrite the action integral in eq. (19) as an integral over the four sphere. We begin by noting that eq.(19) has the following general form,

$$
S_{\zeta}=-\int_{0}^{\infty} \rho^{2} d \rho \int_{-\infty}^{\infty} \frac{d \zeta}{\zeta^{4}} f(\rho, \zeta)
$$

where $f(\rho, \zeta)$ is an analytic function of two variables. Let us split this integral into the sum of the three terms: the integrals over positive and negative $\zeta$ and the integral near the singular point $\zeta=0$. Let us change variables in the first of these integrals,

$$
\int_{0}^{\infty} \rho^{2} d \rho \int_{0}^{\infty} \frac{d \zeta}{\zeta^{4}} f(\rho, \zeta)=\int_{0}^{\pi} d \psi \int_{0}^{\infty} d s \operatorname{sh}^{3} s \sin ^{2} \psi \tilde{f}(\psi, s)
$$

where

$$
\tilde{f}(\psi, s)=f\left(\frac{\sin \psi \operatorname{sh} s}{\operatorname{ch} s-\cos \psi \operatorname{sh} s}, \frac{1}{\operatorname{ch} s-\cos \psi \operatorname{sh} s}\right)
$$

The second term may be written in a similar way,

$$
\int_{0}^{\infty} \rho^{2} d \rho \int_{-\infty}^{0} \frac{d \zeta}{\zeta^{4}} f(\rho, \zeta)=\int_{0}^{\pi} d \psi \int_{0}^{\infty} d \hat{s} \operatorname{sh}^{3} \hat{s} \sin ^{2} \psi f\left(\frac{\sin \psi \operatorname{sh} \hat{s}}{\operatorname{ch} \hat{s}-\cos \psi \operatorname{sh} \hat{s}},-\frac{1}{\operatorname{ch} \hat{s}-\cos \psi \operatorname{sh} \hat{s}}\right)
$$


This expression can be further rewritten as follows (cf. Section 4),

$$
-\int_{0}^{\pi} d \psi \int_{-i \pi}^{-i \pi+\infty} d s \operatorname{sh}^{3} s \sin ^{2} \psi \tilde{f}(\psi, s)
$$

Hence, the integration over positive and negative $\zeta$ corresponds to the integration of one and the same analytical function $\tilde{f}$ along the rays in the complex $s$-plane, $[0, \infty)$ and $(\infty-i \pi,-i \pi]$, respectively. To obtain the complete integral (26)), one has to close the contour. It is clear from eq. (22) that $\zeta$ is small when $s$ is large, so an interval $\left[s_{1}, s_{1}+i \pi\right]$ at large real $s_{1}$ corresponds to a path above the singularity $\zeta=0$ in the complex $\zeta$-plane, i.e., exactly the path that is understood in the integral (26). Hence, the contour has to be closed at infinity, and the action $S_{\zeta}$ is equal to the following expression,

$$
S_{\zeta}=-\int_{0}^{\pi} d \psi \int_{C} d s \operatorname{sh}^{3} s \sin ^{2} \psi \tilde{f}(\psi, s)
$$

where the contour $C$ is shown in fig. 6. This contour can be deformed into an interval

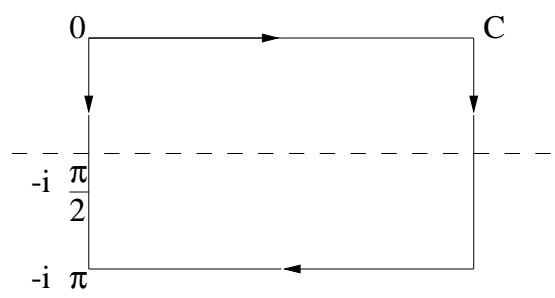

Figure 6:

$[0, \pi]$ on the imaginary axis. The action nicely takes the form of the integral over the four-sphere,

$$
S_{\zeta}=-\int_{0}^{\pi} d \psi \int_{0}^{\pi} d \sigma \sin ^{3} \sigma \sin ^{2} \psi \tilde{f}(\psi,-i \sigma)
$$

To complete the transition to the four-sphere, we have to reformulate the field equations and boundary conditions imposed on the classical solution (see the end of Section 3 ). The former step is easy: one has just to find the explicit form of the function $\tilde{f}$ entering eq. (28) that is obtained from eq. (19) after the change of variables,

$$
\tilde{f}(\psi,-i \sigma)=-\int_{0}^{\pi} d \theta \sin \theta \int_{0}^{2 \pi} d \varphi\left(\frac{\left(\nabla_{S^{4}} \phi\right)^{2}}{2 H^{2}}+\frac{V(\phi)}{H^{4}}\right)
$$


where $\nabla_{S^{4}}$ denotes the gradient on four-sphere. (Note that the gradient and potential terms enter now with the same sign, i.e., the scalar potential has got reversed, cf. refs. [2, 3, 5].) By varying the action, one obtains the standard equation

$$
\Delta_{S^{4}} \phi=\frac{V^{\prime}(\phi)}{H^{2}}
$$

where $\Delta_{S^{4}}$ is the Laplace operator on the four-sphere.

The requirement of real-valuedness of $\phi$ at real $\eta$ (see Section 3) is also easily formulated in terms of the solution on the four-sphere. It is equivalent to the requirement that $\phi$ is real at $s=-i \frac{\pi}{2}+s^{\prime}$ where $s^{\prime}$ is real (see Section 4 ). The latter leads to the relation

$$
\left.\phi\right|_{s=z-i \frac{\pi}{2}}=\left.\phi^{*}\right|_{s=z^{*}-i \frac{\pi}{2}}
$$

Let us make an important assumption that the solution $\phi$ is real on the sphere, i.e., at $s=-i \sigma$. This assumption, together with eq. (31), gives the following condition:

C1. For the solution $\phi$ be real at real $\eta$ it is sufficient and necessary that $\phi$ is symmetric on the sphere with respect to the "equator" $\sigma=\frac{\pi}{2}$.

The requirement that $\phi$ vanishes as $\zeta$ tends to $\infty$ (see the end of Section 3 ) cannot be formulated in terms of the four-sphere, so one has to leave this condition as

C2. Analytical continuation of the solution from the four-sphere to the domain of real $s$ tends to zero as $s$ tends to infinity and $\psi$ tends to zero as $\psi \sim e^{-2 s}$.

To construct the solution on the sphere, let us introduce new angular variables $\sigma^{\prime}, \psi^{\prime}$ that are related to $\sigma, \psi$ as follows,

$$
\cos \sigma^{\prime}=\sin \sigma \cos \psi, \quad \sin \sigma^{\prime} \cos \psi^{\prime}=\cos \sigma
$$

This change of variables corresponds to the shift of the north pole to the point $(\sigma=$ $\left.\frac{\pi}{2}, \psi=0\right)$. In terms of the new variables, the solution has to be symmetric with respect to the meridian line $\psi^{\prime}=\frac{\pi}{2}$. Let us assume, following Coleman and De Luccia, that $\phi$ has greater symmetry, namely, that it is $O(4)$-symmetric. Then $\phi$ depends on one variable $\sigma^{\prime}$ only. Making use of this assumption, one simplifies the expresion for the action,

$$
S_{\zeta}=2 \pi^{2} \int_{0}^{\pi} d \sigma^{\prime} \sin ^{3} \sigma^{\prime}\left(\frac{1}{2 H^{2}}\left(\frac{d \phi}{d \sigma^{\prime}}\right)^{2}+\frac{V(\phi)}{H^{4}}\right)
$$

and obtains the equation for $\phi$,

$$
\frac{d^{2} \phi}{d \sigma^{\prime 2}}+\frac{3}{\operatorname{tg} \sigma^{\prime}} \frac{d \phi}{d \sigma^{\prime}}-\frac{V^{\prime}(\phi)}{H^{2}}=0
$$


The conditions that are imposed on $\phi$ are:

a)

$$
\left.\frac{d \phi}{d \sigma^{\prime}}\right|_{\sigma^{\prime}=0}=\left.\frac{d \phi}{d \sigma^{\prime}}\right|_{\sigma^{\prime}=\pi}=0
$$

(this follows from the requirement of smoothness of $\phi$ );

b) $\phi$ is not constant on the sphere (this is needed to satisfy $\mathrm{C} 2$, see below);

c) $\phi\left(\sigma^{\prime}=0\right)$ is close to the false vacuum, i.e., to $\phi=0 ; \phi\left(\sigma^{\prime}=\pi\right)$ is close to the true vacuum, i.e., to $\phi_{+}$.

Solutions with these properties exist for wide class of the scalar potentials $V(\phi)$ which obey (see refs. [5, Ø, 12])

$$
\left|\frac{V^{\prime \prime}\left(\phi_{0}\right)}{H^{2}}\right|>4
$$

On the other hand, if this inequality does not hold, the condition C2 cannot be satisfied [7, 12. The latter case will be considered in the next Section, and here we assume that the non-trivial solution does exist. The solution under discussion obeys $\mathrm{C} 1$ automatically due to the $O(4)$-symmetry. We postpone the discussion of $\mathrm{C} 2$, and study now the continuation of $\phi$ into the region of real $\eta$.

At this stage, it is convenient to regard $\phi$ as a function of the variable $\mu=$ $\cos \sigma^{\prime}$ rather than of $\sigma^{\prime}$ itself. According to eq. (32), $\mu$ is expressed in terms of the coordinates introduced in Section 4,

$$
\mu=\sin \sigma \cos \psi=i \operatorname{sh} s \cos \psi=\operatorname{chs}^{\prime} \cos \psi
$$

We show in fig. 7 the lines of constant $\mu$ in cooordinates $\left(s^{\prime}, \psi\right)$ (we display the region $\cos \psi<$ ths that corresponds to the physical de Sitter space) By comparing figs. 7 and

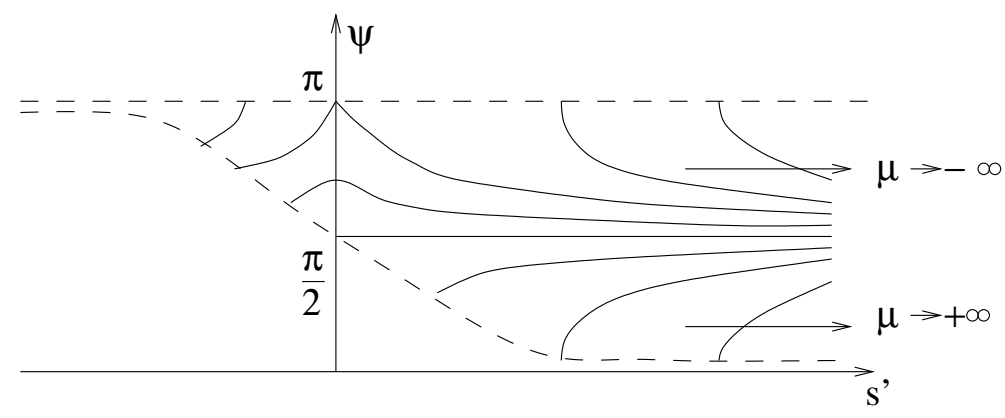

Figure 7: 


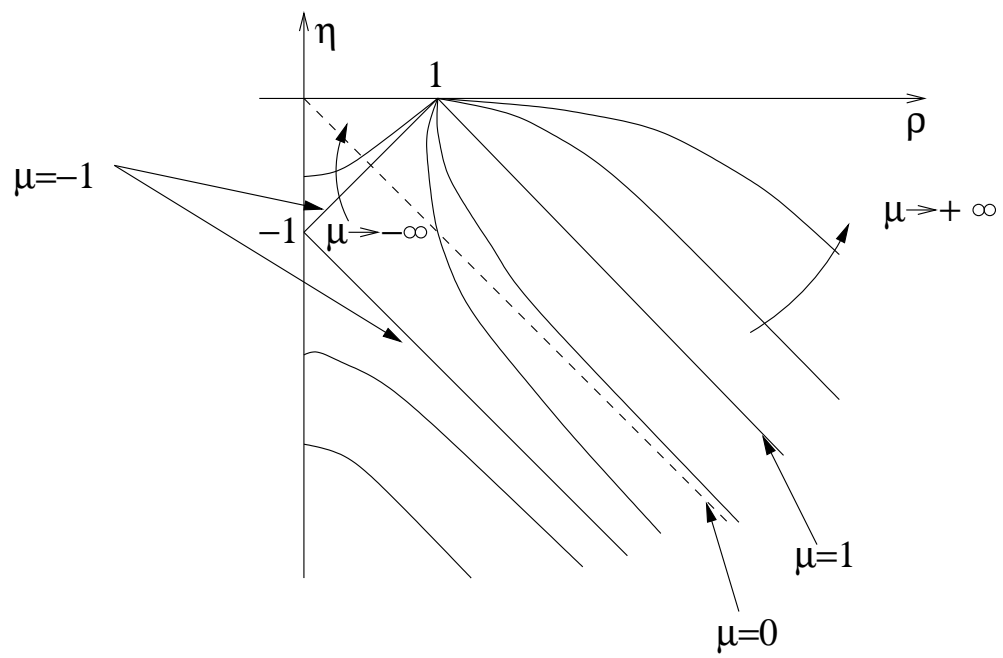

Figure 8:

5, we obtain the set of lines of constant $\mu$, and hence $\phi$, on the plane $(\eta, \rho)$, see fig. 8. It is clear from fig. 8 that $\lim _{\eta \rightarrow 0^{-}, \rho>1} \phi=\lim _{\mu \rightarrow+\infty} \phi$, and $\lim _{\eta \rightarrow 0^{-}, \rho<1} \phi=\lim _{\mu \rightarrow+\infty} \phi$. So, to understand the behavior of $\phi$, one has to consider its asymptotics at $\mu \rightarrow \pm \infty$.

As $\mu$ tends to $+\infty$, the variable $\sigma^{\prime}$ behaves as follows, $\sigma^{\prime}=i \sigma^{\prime \prime}, \sigma^{\prime \prime}$ is real and tends to $+\infty$. In the regime $\mu \rightarrow-\infty, \sigma^{\prime}$ equals $\pi+i \sigma^{\prime \prime}$ where $\sigma^{\prime \prime}$ is again real and tends to $+\infty$. The field equations in these regions are obtained by analytically continuing eq. (34):

$$
\frac{d^{2} \phi}{d \sigma^{\prime \prime 2}}+\frac{3}{\operatorname{th} \sigma^{\prime \prime}} \frac{d \phi}{d \sigma^{\prime \prime}}+\frac{V^{\prime}(\phi)}{H^{2}}=0
$$

The initial conditions that supplement eq. (38) in the two cases are as follows:

$\left.\phi\right|_{\sigma^{\prime \prime}=0}$ is close to 0 as $\mu \rightarrow+\infty$

$\left.\phi\right|_{\sigma^{\prime \prime}=0}$ is close to $\phi_{+}$as $\mu \rightarrow-\infty$

$\left.\frac{d \phi}{d \sigma^{\prime \prime}}\right|_{\sigma^{\prime \prime}=0}=0$ in both cases.

Equation (38) may be viewed as an equation for a particle with coordinate $\phi$ in time $\sigma^{\prime \prime}$. This particle moves classically in the potential $\frac{V(\phi)}{H^{2}}$ with time-dependent friction coefficient $\frac{3}{t h \sigma^{\prime \prime}}$. Since the friction coefficient is larger that 3 at all times, the particle moving with zero initial velocity will always reach the closest minimum of the potential, provided that its initial position is different from $\phi_{0}$. In the former and latter cases this will be $\phi=0$ and $\phi=\phi_{+}$, respectively, see fig. 9 .

Therefore, $\phi \rightarrow 0$ as $\mu \rightarrow+\infty$, while $\phi \rightarrow \phi_{+}$as $\mu \rightarrow-\infty$. This means that

$$
\left.\phi\right|_{\eta \rightarrow 0^{-}, \rho>1}=0,\left.\quad \phi\right|_{\eta \rightarrow 0^{-}, \rho<1}=\phi_{+}
$$




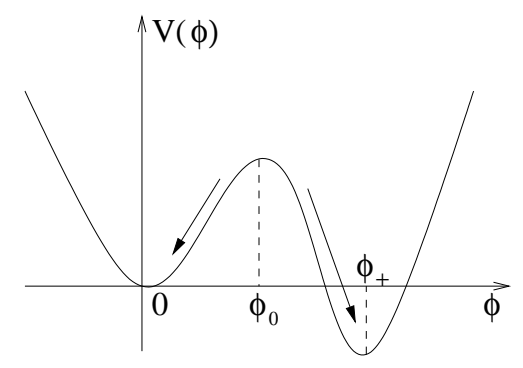

Figure 9:

It follows from the latter relations that the solution describes a bubble of the true vacuum in the false one, whose coordinate size is asymptotically equal to $\rho=1$. The bubbles of other sizes are obtained from this solution by scaling of coordinates, i.e., by the change $\eta \rightarrow \alpha \eta, \rho \rightarrow \alpha \rho$; the leading semiclassical exponent of their formation is independent of their size as the action does not change under scale transformations.

Let us come back to the condition $\mathrm{C} 2$. The behavior of the coordinates $s$ and $\psi$ that enters this condition corresponds, according to eq. (37), to the following behavior of the variable $\mu$,

$$
\mu \sim i e^{s} \cos e^{-2 s} \sim i e^{s}, \quad s \rightarrow \infty
$$

What remains to be done is to analytically continue $\phi$ into the region of large $\mu$. Some care must be taken when performing this continuation, though. It is convenient to recall that the continuation from the region of real $\eta$ to purely imaginary $\eta$ (and, hence, real $\zeta$ ) is to be done as shown in fig. 3. This means that $s$ changes from $s_{1}-i \frac{\pi}{2}$ to $s_{1}$, where $s_{1}$ is real and large (recall the considerations that have lead to eq. (27)). The variable $\mu$ remains in the first quadrant, being far away from the origin, see fig. 10. With this continuation one has $\lim _{\mu \rightarrow+i \infty} \phi=\lim _{\mu \rightarrow+\infty} \phi=0$, so the

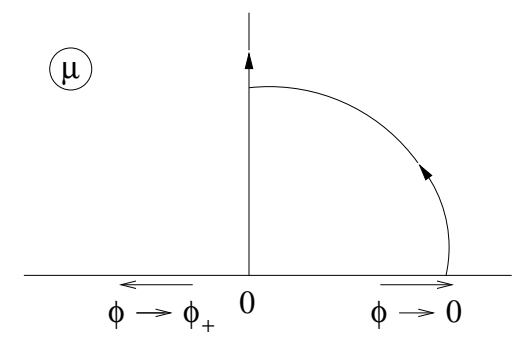

Figure 10:

condition $\mathrm{C} 2$ is indeed satisfied. 
We conclude that the Coleman-De Luccia instanton indeed describes the false vacuum decay in the de Sitter space-time with the qualification that the quantum fluctuations of the scalar field are initially in the state of conformal vacuum.

\section{Hawking-Moss instanton as the limit of con- strained instantons}

Let us now consider the case when a non-trivial solution of the field equation on the four-sphere does not exist. To obtain the false vacuum decay rate, we have to modify our previous arguments. The main idea is that instead of true solution to the field equation, one considers a family of almost saddle point configurations $\phi_{\varepsilon}(\mathbf{x}, \eta)$ which obey the boundary conditions of Section 3 and contribute to the path integral. The action on these configurations depends on the parameter $\varepsilon$, and their contribution to the decay rate tends to maximum as $\varepsilon \rightarrow 0^{+}$. The limiting configuration $\phi_{\varepsilon=0}$ (Hawking-Moss instanton) does not obey the boundary conditions (even though it is a solution to the field equation); however, its action determines the semiclassical exponent of the decay. The situation in this case resembles one that emerges in the study of instanton effects in four-dimensional Yang-Mills-Higgs theories [16].

Let us introduce "unity" in the original path integral (7),

$$
1=\int_{-\infty}^{\infty} d \varepsilon \delta(\varepsilon-\Delta)=\int_{-\infty}^{\infty} d \varepsilon \int_{-\infty}^{\infty} \frac{d \alpha}{2 \pi} e^{i \alpha(\varepsilon-\Delta)}
$$

where

$$
\Delta=\int \frac{d^{3} x d \eta}{2 H^{2} \eta^{2}}\left(\left(\frac{\partial \phi_{1}}{\partial \eta}\right)^{2}+\left(\frac{\partial \phi_{2}}{\partial \eta}\right)^{2}-\left(\frac{\partial \phi_{1}}{\partial x^{i}}\right)^{2}-\left(\frac{\partial \phi_{2}}{\partial x^{i}}\right)^{2}\right)
$$

we now change the order of integration, and integrate over $d \varepsilon d \alpha$ in the very end. After repeating, with slight modifications, the arguments of Sections 3 and 5, we obtain for the decay rate

$$
\Gamma=\int d \varepsilon \exp \left(-S_{\zeta}\left[\phi_{\varepsilon}\right]\right)
$$

where $S_{\zeta}$ is still defined by eq. (33), while the classical solutions $\phi_{\varepsilon}$ obey, besides all conditions of Section 5, an extra constraint,

$$
\frac{2 \pi^{2}}{H^{2}} \int_{0}^{\pi} d \sigma^{\prime} \sin ^{3} \sigma^{\prime} \frac{1}{2}\left(\frac{\partial \phi_{\varepsilon}}{\partial \sigma^{\prime}}\right)^{2}=\varepsilon
$$


To obtain the equation for $\phi_{\varepsilon}$ one subtracts from the action (33) the constraint (42) with the Lagrange multiplier $\lambda$,

$$
\begin{aligned}
S_{\zeta} & -\lambda \frac{2 \pi^{2}}{H^{2}} \int_{0}^{\pi} d \sigma^{\prime} \sin ^{3} \sigma^{\prime} \frac{1}{2}\left(\frac{\partial \phi_{\varepsilon}}{\partial \sigma^{\prime}}\right)^{2} \\
& =\frac{2 \pi^{2}}{H^{2}}(1-\lambda) \int_{0}^{\pi} d \sigma^{\prime} \sin ^{3} \sigma^{\prime}\left(\frac{1}{2}\left(\frac{\partial \phi_{\varepsilon}}{\partial \sigma^{\prime}}\right)^{2}+\frac{V\left(\phi_{\varepsilon}\right)}{H^{2}(1-\lambda)}\right)
\end{aligned}
$$

and varies this expression with respect to $\phi_{\varepsilon}$. The resulting equation is

$$
\frac{d^{2} \phi_{\varepsilon}}{d \sigma^{\prime 2}}+\frac{3}{\operatorname{tg} \sigma^{\prime}} \frac{d \phi_{\varepsilon}}{d \sigma^{\prime}}-\frac{V^{\prime}\left(\phi_{\varepsilon}\right)}{H^{2}(1-\lambda)}=0
$$

We find that, as far as the field equation is concerned, the effect of the constraint (42) is the modification of the scalar potential.

The boundary problem consisting of equation (44), the constraint (42) and conditions (a), (b) and (c) of section 5 has a solution at every positive and sufficiently small $\varepsilon$. To see this, we note that the solution obeying eq. (44) and conditions (a), (b), (c) exists, provided that

$$
\left|\frac{V^{\prime \prime}\left(\phi_{0}\right)}{H^{2}(1-\lambda)}\right|>4
$$

(cf. eq. (36)). At

$$
(1-\lambda) \rightarrow\left|\frac{V^{\prime \prime}\left(\phi_{0}\right)}{4 H^{2}}\right|
$$

this solution tends to a constant on the four-sphere; the integral of its gradient squared tends to zero, so the constraint (42) is indeed satisfied with an appropriate choice of $\lambda$.

From the results of Section 5 it follows that at a given $\varepsilon>0$, the analytical continuation of the solution $\phi_{\varepsilon}$ to physical space-time describes the bubble of the true vacuum. On the other hand, as $\varepsilon \rightarrow 0^{+}$, the configuration $\phi_{\varepsilon}$ tends to a constant field on the four-sphere, $\phi=\phi_{0}$, i.e., it becomes the Hawking-Moss instanton. It is known [7, 12] that in the models under discussion, this instanton is an absolute minimum of the action $S_{\zeta}$, at least among the $O(4)$-symmetric configurations. Hence, $S_{\zeta}\left[\phi_{\varepsilon}\right]$ as a function of $\varepsilon$ has a minimum at $\varepsilon=0$, so the largest contributions into the rate (41) come from the solutions $\phi_{\varepsilon}$ at small $\varepsilon$. The integral (41) is then determined by the limiting point $\varepsilon=0$ and is equal to

$$
\Gamma \propto e^{-S_{\zeta}\left[\phi_{0}\right]}
$$


where the exponent is the action of the Hawking-Moss instanton,

$$
S_{\zeta}\left[\phi_{0}\right]=\frac{8 \pi^{2}}{3 H^{4}} V\left(\phi_{0}\right)
$$

Thus, the Hawking-Moss instanton is the limiting case of the constrained instantons, $\phi_{\varepsilon}(\mathbf{x}, \eta)$. The configuration $\phi_{\varepsilon}(\mathbf{x}, \eta)$ at $\varepsilon>0$ has the same structure as the Coleman-De Luccia instanton considered in the previous Section. We conclude that the false vacuum decay again occurs through the formation of bubbles of the new phase.

\section{$\begin{array}{ll}7 & \text { Jumps up }\end{array}$}

Finally, let us discuss an interesting property of the scalar field in the de Sitter spacetime which is absent in the case of flat space. In the de Sitter space-time, there is a possibility of tunneling transitions from true vacuum to false one; the possibility of "jumps up" due to quantum fluctuations of the scalar field was pointed out in ref. [17] in the context of inflationary theories. The energy needed for these transitions comes from the gravitational field; within the approximation made in this paper, the latter represents, in fact, an infinite source of energy. The description of these transitions is natural in the framework advocated in this paper.

Indeed, when introducing the coordinates $\sigma^{\prime}, \psi^{\prime}$ (see Section 5) we could write, instead of eq. (32), another change of variables,

$$
\cos \sigma^{\prime}=\sin \sigma \cos \left(\psi-\psi_{0}\right), \quad \sin \sigma^{\prime} \cos \psi^{\prime}=\cos \sigma
$$

where $\psi_{0}$ is a parameter between 0 and $\pi$. This would correspond to the shift of the pole on the four-sphere to the point $\sigma=\frac{\pi}{2}, \psi=\psi_{0}$. There again exists an $O(4)$ symmetric solution of eq. (30) in this coordinate system, that depends on $\sigma^{\prime}$ only and coincides, as the function of $\sigma^{\prime}$, with the solution considered in Section 5. (We discuss here the case when the non-trivial solution of Coleman-De Luccia type exists; the considerations that follow are straightforwardly generalized to the Hawking-Moss case.) However, this solution does not make sense at arbitrary $\psi_{0}$. This is clear from the fact that its analytical continuation to real $\rho$ and $\eta$ does not obey the smoothness property $\left.\frac{\partial \phi}{\partial \rho}\right|_{\rho=0}=0$. Indeed, let us introduce, as in Section 5 , the variable $\mu=\cos \sigma^{\prime}$, which is now related to $s^{\prime}$ and $\psi$ by

$$
\mu=\operatorname{ch} s^{\prime} \cos \left(\psi-\psi_{0}\right)
$$


(cf. eq. (37)). Making use of eq. (23), we obtain

$$
\left.\frac{\partial \phi}{\partial \rho}\right|_{\rho=0}=\left.\frac{e^{s^{\prime}}}{\operatorname{ch} s^{\prime}} \frac{\partial \phi}{\partial \psi}\right|_{\psi=\pi}=-\left.\operatorname{ch}^{\prime} \sin \left(\pi-\psi_{0}\right) \frac{d \phi}{d \mu}\right|_{\mu=c h s^{\prime} \cos \left(\pi-\psi_{0}\right)}
$$

which is not equal to zero if $\psi_{0} \neq 0, \pi$. Hence, the solution can describe a physical process only if $\psi_{0}$ equals 0 or $\pi$. The former possibility has been considered in Section 5. Let us now turn to the case $\psi_{0}=\pi$. Instead of eq. (37) we have in this case

$$
\mu=-i \operatorname{sh} s \cos \psi=-\operatorname{ch}^{\prime} \cos \psi
$$

Hence (cf. eq. (39))

$$
\lim _{\eta \rightarrow 0^{-}, \rho>1} \phi=\lim _{\mu \rightarrow-\infty} \phi=\phi_{+}, \quad \lim _{\eta \rightarrow 0^{-}, \rho<1} \phi=\lim _{\mu \rightarrow+\infty} \phi=0, \quad \lim _{s \rightarrow \infty, \phi \sim e^{-2 s}} \phi=\phi_{+}
$$

These are precisely the properties that one would anticipate for the transitions from the true vacuum to the false one. This means that the solution with $\psi_{0}=\pi$ describes the formation of a bubble of the false vacuum in the true one. The probability of this process is again determined by the action (33), but now one has to subtract the action of the trivial solution corresponding to the true vacuum,

$$
\frac{2 \pi^{2}}{H^{4}} \int_{0}^{\pi} d \sigma^{\prime} \sin ^{3} \sigma^{\prime} V\left(\phi_{+}\right)=\frac{8 \pi^{2}}{3 H^{4}} V\left(\phi_{+}\right)
$$

In this way we obtain the probability of the bubble formation,

$$
\Gamma \propto \exp \left[-S_{\zeta}+\frac{8 \pi^{2}}{3 H^{4}} V\left(\phi_{+}\right)\right]
$$

The limiting case of flat space-time is obtained by sending the Hubble parameter to zero. The action $S_{\zeta}$ tends to a constant in this limit, this constant being the exponent of the false vacuum decay in flat space. Since $V\left(\phi_{+}\right)<0$, the probability of the transition from the true vacuum to the false one vanishes in this limit, as expected.

\section{Conclusions}

In this paper, the false vacuum decay in the de Sitter space-time was discussed within the path integral approach. We considered the theory of one scalar field and chose 
conformal vacuum of the scalar particles above the false classical vacuum as a definition of the quantum false vacuum. In this case we reproduced from the first principles the Coleman-De Luccia and Hawking-Moss results for the leading semiclassical exponents of the decay rate.

We gave the interpretation of the Hawking-Moss instanton as a limiting case of constrained instantons and observed that in spite of the fact that it determines the transition probability, the structure of the field after tunneling is in fact determined by other configurations that are close to this instanton on the four-sphere. The result of the tunneling process is, as usual, the bubble of the true vacuum.

We discussed yet another interesting phenomenon, namely, the transitions from the true vacuum to the false one, and calculated the probability of this process. This probability tends to zero in the limit of flat space-time, in accord with expectations.

Though we concentrated in this paper on a particular class of processes, our approach that explicitly accounts for the initial state of the quantum field (in our case it was the conformal vacuum) is straightforward to generalize to other semiclassical processes in curved space-time. The key ingredients of our approach - the boundary conditions (13) and (14) - are in fact quite general, provided the positive-frequency conditions are understood in terms of asymptotic properties of the solution $\phi(x)$ with respect to the set of modes appropriate to a given vacuum of the quantum field. It is another matter that the concrete analytical continuations of the solution into the domain of complex coordinates, which were used in this paper, are likely to be specific to the de Sitter space and to the conformal vacuum of scalar particles.

As a final remark, let us point out that there are choices of coordinates in the de Sitter space-time that differ from eq. (2) and, correspondingly, the choices of the vacuum of the scalar particles that differ from the conformal vacuum. In particular, Derulle [18] considered the false vacuum decay, in the background metrics approximation, making use of the static coordinate system, and reproduced the Coleman-De Luccia and Hawking-Moss results as well. On the other hand, the vacuum defined with respect to the static coordinate system is not invariant even under translations [9] and, therefore, it is quite different from the vacuum considered in this paper. So, the coincidence of the results of our paper and ref. [18] appears surprizing. It is worth noting, however, that the quantum vacuum was not explicitly defined in ref. [18]. We think that this coincidence may be explained as follows: in effect, the analysis of ref. 18] applies not to tunneling from the vacuum of the static coordinate system, but to transitions from the conformal vacuum which looks as a highly excited state from the 
point of view of the static coordinate system. This point deserves further analysis.

We hope that this paper clarifies certain aspects of the path integral fomalism in curved space-time and opens up new ways of its application to various semiclassical processes.

\section{References}

[1] M.B. Voloshin, I.Yu. Kobzarev, L.B. Okun, Yad. Fiz., v. 20, p. 1229, 1974.

[2] S. Coleman, Phys. Rev., v. D15, p.2929, 1977.

[3] C.G. Callan, S. Coleman, Phys. Rev., v. D16, p.1792, 1977.

[4] S. Coleman, V. Glaser, A. Martin, Comm. Math. Phys. v.58 p. 211, 1978

[5] S. Coleman, F. De Luccia, Phys. Rev., v. D21, p.3305, 1980.

[6] A.H. Guth, E. Weinberg, Nucl. Phys., v. B212, p. 321, 1983.

[7] S.W. Hawking, I.G. Moss, Nucl. Phys., v. B224, p. 180, 1983

[8] A.A. Grib, S.G. Mamayev, V.M. Mostepanenko, Quantum effects in intense external fields. Moscow: Atomizdat, 1980

[9] N.D. Birrell, P.C.W. Davies, Quantum fields in curved space. Cambridge: Cambridge Univ. Press, 1982

[10] A.D. Linde, Nucl. Phys., v. B216, p. 421, 1983.

[11] A.A. Starobinsky, in: Fundamental interactions. Moscow: MGPI, 1984, p. 55

[12] A.S. Goncharov, A.D. Linde, Elem. Chast. At. Yad. v. 17, p. 837, 1986.

[13] S.W. Hawking, G.F.R. Ellis, The large scale structure of space-time. Cambridge: Cambridge Univ. Press, 1973

[14] A.D. Linde, Physics of elementary particles and inflationary cosmology. Moscow: Nauka, 1990

[15] V.A. Rubakov, D.T. Son, P.G. Tinyakov, Phys. Lett. v. B278, p. 279, 1992;

A.N. Kuznetsov, P.G. Tinyakov, Phys. Rev., v. D56, p.1156, 1997. 
[16] I. Affleck, Nucl. Phys., v. B191, p. 429, 1981.

[17] A.D. Linde, Phys. Lett., v. B131, p. 330, 1983.

[18] N. Deruelle, Mod. Phys. Lett., v. A4, p. 1297, 1989. 